УДК 622.235 .5

\title{
УСЛОВИЯ ФОРМИРОВАНИЯ ВОЛН НАПРЯЖЕНИЯ ПРИ ВЗРЫВЕ ШПУРОВЫХ ЗАРЯДОВ ВЗРЫВЧАТОГО ВЕЩЕСТВА И ИХ ВЛИЯНИЕ НА КАЧЕСТВО РАЗРУШЕНИЯ ГОРНЫХ ПОРОД ПРИ ПРОХОДКЕ ГОРНЫХ ВЫРАБОТОК
}

\author{
Масаев Юрий Алексеевич1, \\ myua.spssh@kuzstu.ru
}

\author{
Масаев Владислав Юрьевич1, \\ masaevvu@kuzstu.ru
}

Дрозденко Юрий Вадимович1,2, duv.gmik@kuzstu.ru

\author{
Аксенова Олеся Юрьевна ${ }^{1}$, \\ aka.gp061@kuzstu.ru \\ 1 Кузбасский государственный технический университет имени Т.Ф. Горбачева, \\ Россия, 650000, г. Кемерово, ул. Весенняя, 28. \\ 2 Филиал Кузбасского государственного технического университета имени Т.Ф. Горбачева в г. Прокопьевске, \\ Россия, 650000, г. Прокопьевск, ул. Ноградская, 19 а.
}

Актуальность. При разработке месторождений полезных ископаемых взрывным способом требуется разрушать горные породы, залегающие на различных глубинах в различных горно-геологических условиях и имеющих различные фризикотехнические свойства. Взрыв шпуровых зарядов взрывчатого вещества формирует волны напряжений и давление продуктов взрыва, которые по-разному действуют на разные горные породы, и для улучшения их действия шпуровые заряды необходимо взрывать с различным замедлением.

Цель: более полный и качественный учет энергетических характеристик взрывчатых веществ, положения шпуровых зарядов относительно естественной трещиноватости горной породы, крепости вмещающих горных пород, сечения горных выработок для повышения качества разрушения взрываемых горных пород.

объекты: объекты подземного строительства, угольные и рудные шахты, горные предприятия по добыче полезных ископаемых открытым и подземным способом.

Методы. Для достижения поставленной цели использовались натурные исследования, позволившие установить зависимость качества разрушения горной породы от времени срабатывания шпуровых зарядов, а также аналитические исследования, позволившие определить значения предельных радиусов расширения зарядной полости для некоторых типов взрывчатых веществ.

Результаты. Результатами исследования являются более полный учет энергетических характеристик взрывчатых веществ, оптимальное объемное положение шпуровых зарядов относительно систем естественной трещиноватости среды, корреляция их с крепостью горных пород, сечением горных выработок и глубиной заложения зарядов взрывчатых веществ, приводящими к созданию рациональных объемов врубов, обеспечивающих наиболее высокие показатели взрывных работ.

\section{Ключевые слова:}

Волны напряжений, детонация взрывчатого вещества, трещиноватость, врубовые шпуры, короткое замедление, коэффрициент использования шпуров, неэлектрическая система инициирования, капсюль-детонатор.

\section{Введение}

При проведении горных выработок с применением буровзрывных работ эффективность разрушения горной породы во многом зависит от эффективности действия зарядов взрывчатого вещества (BВ) врубовых шпуров. Четкость проработки и достаточная ширина плоскости обнажения во врубовой полости оказывает важное влияние на величину коэффициента использования шпуров и на общие качественные и количественные показатели эффективности взрыва $[1,2]$.

Одной из важнейших величин, определяющих параметры методов управления энергией взрыва, является продолжительность разрушения породы действием взрыва. Прямое влияние на продолжительность процессов разрушения взрываемой горной по- роды при короткозамедленном взрывании оказывает выбор величины замедления срабатываемых зарядов $\mathrm{BB}$. Взаимодействие зарядов ВВ при взрывании горных пород с коэффициентом крепости $f=1-14$, относящихся по акустической жесткости ко второй группе, должно рассматриваться с учетом двух действующих компонентов - волн напряжения и давления продуктов детонации. В начальной стадии процесса разрушения основную роль играют продукты взрыва, под действием которых породный массив подвергается пластическим деформациям, и за счет этого происходит увеличение начального объема зарядной полости до тех пор, пока давление продуктов детонации не достигнет временного предела прочности породы при объемном сжатии, которое в 10-15 раз больше, чем при одноосном сжатии [3-7]. 


\section{Методика определения предельных радиусов}

Закон сдвижения «продукты детонации - разрушаемая порода» описывается уравнением:

$$
\frac{d u^{2}}{d r}+\frac{3 u^{2}}{r}=\frac{2 \rho_{1}}{\rho_{a}} \cdot \frac{r_{k}^{3 \gamma}}{r^{3(\gamma+1)}}-\frac{2 \rho_{a}}{\rho_{a} \cdot r},
$$

решением которого является выражение, позволяющее определить движение границы раздела для любого момента времени:

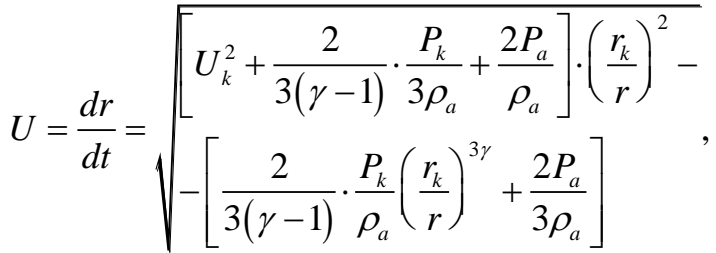

где $P_{a}$ - атмосферное давление; $\rho_{a}$ - плотность среды; $\gamma$ - показатель изэнтропы; $r$ - радиус полости; $P_{k}-$ давление продуктов взрыва; $r_{k}$ - радиус полости в любой точке массива; $U$ - скорость движения границы раздела.

Для типичных ВB расширение продуктов взрыва до давления $P=2000 \kappa г / \mathrm{cm}^{3}$ происходит по закону $P_{0} V_{0}^{3}=P V^{3}$ или $P V^{3}=$ const, а затем, при более низких давлениях, $P V^{\gamma}=$ const.

Сопряжение этих двух законов приводит к зависимости:

$$
\frac{V_{\text {пр }}}{V_{\text {нач }}}=\left(\frac{P_{0}}{P_{k}}\right)^{1 / 3} \cdot\left(\frac{P_{k}}{P_{a}}\right)^{1 / 3},
$$

где $V_{\text {нач }}-$ начальный объем продуктов детонации; $V_{\text {пр }}$ - конечный объем продуктов детонации.

В таблице приведены вычисленные значения предельных радиусов расширения зарядной полости для аммонитов АП-5ЖВ и 6ЖВ $[8,9]$.

Таблица. Значения предельных радиусов расширения зарядной полости

Table. Values of limiting expansion radii of the charging cavity

\begin{tabular}{|c|c|c|}
\hline \multirow{2}{*}{$\begin{array}{c}\text { Коэффициент крепости } \\
\text { горной породы, } f \\
\text { Rock hardness } \\
\text { coefficient, } f\end{array}$} & \multicolumn{2}{|c|}{$\begin{array}{c}\text { Тип взрывчатого вещества } \\
\text { Explosive type }\end{array}$} \\
\cline { 2 - 3 } & $\begin{array}{c}\text { Aммонит АП-5ЖВ } \\
\text { AP-5ZhV ammonite }\end{array}$ & $\begin{array}{c}\text { Аммонит 6ЖВ } \\
\text { 6ZhV ammonite }\end{array}$ \\
\hline $4-6$ & 2,0 & 2,14 \\
\hline $6-8$ & 1,9 & 2,0 \\
\hline $8-12$ & 1,7 & 1,8 \\
\hline $12-14$ & 1,6 & 1,7 \\
\hline
\end{tabular}

При детонации взрывчатого вещества в горной породе сразу же зарождается волна напряжений $[8,9]$. Давление во фронте волны напряжения при ее перемещении по массиву породы определяется приближенно соотношением

$$
\sigma_{r}=P_{0}\left(\frac{r_{0}}{r_{x}}\right)^{n},
$$

где $\rho_{r}$ - радиальное напряжение во фронте волны напряжений; $r_{x}$ - расстояние до точки, в которой определяется напряжение; $n$ - коэффициент $(n=1-2)$.
Максимальные радиальные напряжения в крепких породах при взрывании удлиненных зарядов ВВ по рекомендации профессора А. Н. Ханукаева можно определить по формуле:

$$
\sigma_{r_{\max }}=\frac{\rho C_{p}}{g}\left(\frac{24}{\bar{r}} \frac{3600}{\bar{r}^{2}} \frac{86400}{\bar{r}^{3}}\right) 10^{2},
$$

где $\rho C_{p}-$ акустическая жесткость породы; $\bar{r}=\frac{r_{x}}{r_{0}}-$ относительное расстояние до определяемой точки.

Величина напряжений на контакте «заряд-порода» может быть вычислена по формуле:

$$
\sigma_{\text {max }}=k_{1} P_{0},
$$

где $k_{1}$ - коэффициент преломления давления из заряда ВВ в горную породу.

$$
k_{1}=\frac{2}{1+\frac{\rho_{\mathrm{BB}} D_{\mathrm{BB}}}{\rho_{\text {пор }} C_{\text {пор }}}},
$$

где $\rho_{\mathrm{BB}}, \rho_{\text {пор }}-$ объемный вес ВВ и горной породы; $D_{\mathrm{BB}}, C_{\text {пор }}-$ скорость детонации ВВ и скорость распространения возмущений в породе.

Эти формулы справедливы для зарядов ВВ, у которых отношение длины к диаметру больше 10-12. Дальнейшее увеличение этого отношения не приводит к увеличению напряжений на фронте волны.

Время формирования волны сжатия может быть определено из выражения:

$$
t_{1}=\frac{2 r}{U}
$$

Под действием прямой волны напряжений, кроме действующих в радиальном направлении напряжений сжатия, возникают тангенциальные растягивающие напряжения, которые зарождают радиальные трещины. Образование трещин под действием тангенциальных напряжений происходит без увеличения объема разрушаемого массива.

При взрыве заряда ВВ нагрузка на окружающую породу прикладывается мгновенно, а снижается медленно, по мере падения давления с уходом газообразных продуктов взрыва из зарядной полости в процессе растрескивания окружающей породы. Вследствие этого по породному массиву вслед за ударной волной распространяется волна разряжения на значительно большую глубину, чем волна сжатия [8-12].

Взрывная волна, дойдя до обнаженной поверхности, отражается от нее с переменой знака, движется вглубь массива. Поскольку давление на переднем фронте взрывной волны значительно выше статического давления газообразных продуктов и в породах с высокой и средней акустической жесткостью растягивающие напряжения в отраженных волнах имеют значительную по величине амплитуду, то отраженная волна вызывает упругие колебания породы.

В породах с низкой акустической жесткостью эти колебания будут иметь значительно меньшую продолжительность и величину амплитуды, и напряженное состояние породы во времени будет в основном 
характеризоваться только изменением статических напряжений $[13,14]$.

Вследствие этого в крепких породах процесс образования трещин связан с действием проходящей прямой волны сжатия и последующими упругими колебаниями при отражении волны от обнаженных поверхностей. В слабых породах зарождение трещин происходит только под воздействием проходящей прямой взрывной волны. Дальнейшее развитие и расширение этих трещин во всех рассматриваемых породах происходит за счет статического давления газов взрыва.

Первая фаза разрушения породы под воздействием волн взрыва может протекать без увеличения объема разрушаемой породы и возможности свободного ее смещения в каком-либо направлении. Дальнейшее развитие и расширение этих трещин не может успешно протекать без увеличения объема разрушаемой породы и возможности свободного ее смещения в каком-либо направлении [15].

Величина замедления между двумя соседними зарядами или сериями зарядов ВВ при короткозамедленном взрывании может быть определена из условия, что первая фаза разрушения породы зарядами последующей серии может протекать параллельно со второй фазой разрушения породы зарядами предыдущей серии. Однако к моменту начала второй фазы разрушения породы последующей серии порода предыдущей серии должна начать отходить, то есть к этому моменту должны быть созданы условия, аналогичные наличию дополнительной обнаженной поверхности, а время замедления должно быть равно продолжительности второй фазы разрушения породы.

При таком замедлении каждая последующая серия зарядов ВВ взрывается еще в породе, имеющей статические напряжения от взрывов зарядов предыдущей серии, и процесс зарождения трещин, их развитие и расширение протекает практически одновременно. В породах слабых и средней крепости первая фаза составляет около $30 \%$ времени разрушения породы взрывом $[8,15,16]$.

На основании экспериментальных исследований по определению оптимального замедления при короткозамедленном взрывании можно сделать вывод, что величина оптимального замедления в различных условиях взрывания неодинакова и имеет прямую связь с продолжительностью процесса разрушения породы взрывом. В породах крепких время замедления равно времени разрушения породы в глубине массива, в породах средней крепости и слабых время замедления меньше, чем продолжительность разрушения породы взрывом, и оно зависит от величины заряда ВВ и глубины шпуров.

С другой стороны, продолжительность разрушения породы при взрыве складывается из суммы времени зарождения и распространения в породном массиве трещин под воздействием давления газов взрыва и увеличение объема разрушения породы происходит только во второй фазе разрушения. Если рассматривать процесс разрушения породы при короткозамедленном взрывании в динамике, то вполне возможно допущение, что первая фаза разрушения породы последующего взрыва может протекать одновременно со второй фазой разрушения породы от взрыва предыдущего заряда ВВ. Отсюда величина замедления между взрывами зарядов ВВ может быть меньше продолжительности полного разрушения породы, но к началу второй фазы разрушения породы данной серии порода предыдущей серии должна отходить от породного массива и не препятствовать развитию второй фазы разрушения, и к этому времени должны быть созданы условия аналогичные наличию дополнительных обнаженных поверхностей.

При замедлении, равном продолжительности второй фазы разрушения породы, каждый последующий взрыв воздействует на породу, находящуюся еще в напряженном состоянии от предыдущего взрыва, и взрывная волна следующего взрыва еще может пройти в породу предыдущей серии, находящуюся в состоянии второй фазы разрушения, и способствовать дополнительному дроблению этой породы. При таких малых замедлениях возможно вторичное дробление породы за счет столкновения разлетающихся масс при пересечении траекторий полета [17].

При взрывании с замедлениями меньшими продолжительности второй фазы разрушения взрыв носит характер мгновенного, порода предыдущих серий будет препятствовать увеличению объема и сдвижению породы последующих серий. С увеличением интервала замедления до величины, обеспечивающей полное формирование дополнительных обнаженных поверхностей, происходит возврат к обычному замедленному взрыванию, так как по времени формирования обнаженных поверхностей в породном массиве динамические и статистические напряжения или исчезают полностью, или имеют незначительную величину. При этом увеличивается разброс взорванной породы, и наблюдается более значительное у забоя скопление газообразных продуктов взрыва, чем при обычном замедленном взрывании. Таким образом, величина интервала замедления в каждом случае взрывания тесно связана с продолжительностью процесса разрушения породы взрывом.

В целях снижения потребных интервалов замедления у электродетонаторов короткозамедленного действия необходимо разрабатывать типовые параметры буровзрывных работ, обеспечивающие высокую эффективность как буровзрывного цикла, так и всего горнопроходческого цикла, а также устанавливать оптимальные величины замедлений для электродетонаторов.

Как уже было отмечено ранее, продолжительность процесса разрушения породного массива зависит от свойств самой породы, величины заряда и свойств применяемых $\mathrm{BB}$, а также от величины линии наименьшего сопротивления. Чтобы сделать способ короткозамедленного взрывания более гибким для всех условий взрывания, необходимо иметь большее число ступеней и интервалов замедления, так как в практике проведения горных выработок применяются параметры взрывных работ, требующие различных интервалов замедления. 


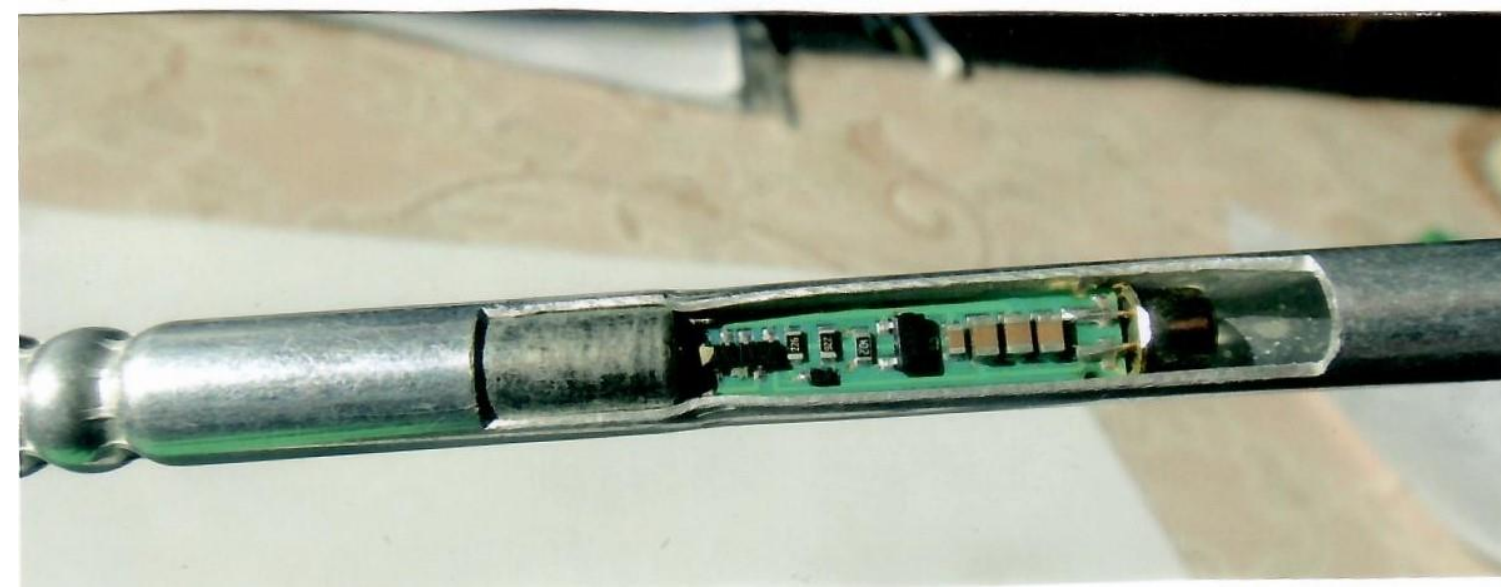

Рисунок. Капсюль-детонатор неэлектрической системы взрывания (СИНВ)

Figure. Non-electric firing system (SINV) primer-detonator

Такие условия взрывания можно осуществлять при использовании неэлектрической системы взрывания (СИНВ), устройства которой (рисунок) служат для трансляции инициирующего сигнала и инициирования боевиков шпуровых зарядов ВВ с заданной временной задержкой широкого диапазона замедления от 0 до 10000 миллисекунд [17].

Капсюль-детонатор представляет собой гильзу из алюминиевого сплава или стали с томпаковым покрытием, внутри которой размещена микросхема, обеспечивающая управление срабатывания замедлительного элемента, инициирующего элемента и основного заряда ВВ. К корпусу детонатора присоединена ударно-волновая трубка (УВТ), которая представляет собой пластиковую трубку, состоящую из нескольких слоев, наружный диаметр равен 3,5 мм. На внутреннюю поверхность трубки нанесен слой взрывчатого материала в количестве 20 мг на 1 м, зажигание которого инициирующим импульсом приводит к образованию устойчивого процесса, распространяющегося внутри трубки со скоростью $2000 \mathrm{~m} / \mathrm{c}$, а трубка остается неповрежденной [17-19].

Применение системы СИНВ предназначено для открытых горных работ и для подземных взрывных работ, они могут использоваться в угольных и рудных шахтах, не опасных по газу и пыли (II категории) Но схема монтажа взрывных сетей более сложна ударно-волновые трубки, выходящие из шпуров, монтируются одновременно с помощью детонирующих шпуров или от устройства СИНВ мгновенного действия и при большом количестве УВТ собираются в пучки и обвязываются двойной петлей детонирующего шнура. Число УВТ в одном пучке (связке) может составлять 15 штук. Все узлы детонирующего шнура (ДШ) соединяют в одну взрывную сеть и ини-

\section{СПИСОК ЛИТЕРАТУРЫ}

1. Масаев Ю.А., Масаев В.Ю. Исследование условий формирования зон трещинообразования в породном массиве при сооружении горных выработок с применением взрывных работ // Вестник научного центра по безопасности работ в угольной промышленности. - 2020. - № 1. - С. 17-22. циируются детонатором, однако такой способ взрывания ведет к увеличению количества взрываемого ДШ в забое, повышает риск подбоя волноводов, приводящего к отказам. При таком способе соединения пучков необходимо выдерживать расстояние до активных частей УВТ более $20 \mathrm{~cm}$, учитывая, что скорость детонации ДШ более чем в 3 раза выше скорости распространения процесса в УВТ $[20,21]$

Учитывая сложность управления энергией взрыва, необходимо в каждом конкретном случае выбирать оптимальные параметры буровзрывных работ, но иногда, пытаясь решить обратную задачу, стараясь подогнать все условия взрывания под интервал замедления. Но не всегда этого удается достичь из-за ограниченной возможности варьирования глубиной шпуров и величиной зарядов ВВ.

\section{Заключение}

Эффективность взрывных работ при проведении горных выработок, как уже было отмечено ранее, в целом по забою зависит от формы, объема и четкости проработки врубовой полости, и для конкретных условий взрывания существуют свои оптимальные объемы врубов, обеспечивающие наиболее высокие показатели взрывных работ. Учет энергетических характеристик взрывчатого вещества, объемного положения шпуровых зарядов относительно систем естественной трещиноватости среды наряду с крепостью горных пород, сечением горных выработок и глубиной заложения взрывных зарядов позволяет добиться оптимальных результатов. В основу расчета удельного заряда взрывчатого вещества должны быть заложены особенности действия взрыва в условиях значительного зажима в трещиноватой среде.

2. Першин В.В., Масаев Ю.А., Мильбергер Н.В. Исследование закономерностей формирования импульса давления в зарядной полости // Известия высших учебных заведений. Горный журнал. - 2015. - № 1. - С. 93-102.

3. Experimental study of rock fragmentation under different stemming conditions in model blasting / Zhang Zong-Xian, Qiao Yang, Chi Li Yuan, Hou De-Feng // International Journal of Rock Mechanics and Mining Sciences. - 2021. - № 143. URL: 
https://www.researchgate.net/publication/351266679_Experimenta 1_study_of_rock_fragmentation_under_different_stemming_condit ions in model blasting (дата обращения: 12.02.2021).

4. Масловский А.Н., Лукьянов А.Н. Актуальность буровзрывных работ с прямыми врубами при проведении горизонтальных горно-разведочных выработок с влиянием геологоструктурных особенностей массива // Известия Томского политехнического университета. - 2014. - Т. 325. - № 1. C. $166-172$.

5. Sarkar K., Singh T.N., Reddy D.V. Prediction of strength parameters by dynamic wave // Int J Earth Sci Eng - 2009. № 2 (1). - P. 12-19.

6. Saikat Kuilia, Vedala Rama Sastry. A numerical modelling approach to assess the behaviour of underground cavern subjected to blast loads // International Journal of Mining Science and Technology. - 2018. - № 28. - P. 975-983.

7. Григоровский И. Использование ГГИС при проектировании буровзрывных работ на открытых горных работах // Золото и технологии. - 2018. - № 2 (40). - С. 50-53.

8. Масаев Ю.А., Карасев В.А., Саяпин В.В. Новые средства инициирования промышленных зарядов ВВ // Безопасность жизнедеятельности предприятий в промышленно развитых регионах: VIII Международная научно-практическая конференция. Кемерово: КузГТУ, 2009. - С. 173-175

9. Масаев Ю.А., Доманов В.П. Электродетонаторы с электронным замедлением. Опыт промышленного применения // Вестник Кузбасского государственного технического университета. - 2010. - № 2. - C. 102-106.

10. Isheyskiy V., Sanchidrián J.A. Prospects of applying MWD technology for quality management of drilling and blasting operations at mining enterprises // Minerals. - 2020. - № 10. DOI https://doi.org/10.3390/min10100925

11. Лукьянов В.Г., Комащенко В.Н., Шмурыгин В.А. Взрывные работы. - Томск: Изд-во Томского политехнического университета, 2008. - 404 с.

12. Взрывоопасность газа при подземной добыче угля в Кузбассе / Е.А. Козловский, Г.Н. Шаров, А.Э. Конторович, Г.И. Грицко, Ф.А. Кузнецов, М.В. Курленя, В.А. Ковалев, В.И. Ростовцев, И.М. Белозеров, В.А. Черноок, В.А. Минин, Н.Ю. Вашлаева // Фундаментальные и прикладные вопросы горных наук. 2018. - T. 5. - № 1. - C. 76-82

13. Методы ведения взрывных работ. Специальные взрывные работы / М.И. Ганопольский, В.Л. Барон, В.А. Белин, В.В. Пуп- ков, В.И. Сивенков. - М.: Изд-во Московского государственного горного университета, 2007. - $563 \mathrm{c}$

14. Перепечаев И.Ф., Кравченко А.Н., Баширов А.В. Совершенствование буровзрывных работ в карьерах объединения // Горный журнал. - 2014. - № 6. - С. 28-31.

15. Обоснование параметров буровзрывной подготовки руд к подземному блочному выщелачиванию / В.И. Ляшенко, О.Е. Хоменко, Б.Н. Андреев, В.И. Голик // Горный информационно-аналитический бюллетень (научно-технический журнал). - 2021. - № 3. - С. 58-71.

16. Velocity amplification of seismic waves through parallel fractures near a free surface in fractured rock: a theoretical study / Ping Zhang, Ering Nordlund, Graham Swan, Changping Yi // Rock Mechanics and Rock Engineering. - 2019. - № 52. - P. 199-213.

17. Андреев В.В., Тимошин И.В. Особенности взрывания неэлектрической системой СИНВ // Взрывное дело. - 2007. - № 97/54. C. 131-134. URL: https://sbornikvd.ru/vd_09754/index.html (дата обращения: 12.02.2021)

18. Реализация программы проведения экспериментальных взрывов в филиалах АО «УК «Кузбассразрезуголь» на период 2020-2021 гг. / С.В. Котин, Д.М. Пархоменко, В.В. Пронин, А.В. Бервин // Горная Промышленность. - 2020. - № 5. C. $44-46$.

19. Alaattin Sakcali, Huseyin Yavuz, Halim Cevizci. Developing a computer software and an android application for bench blasting // 6th International Conference on Computer Applications in the Minerals Industries (CAMI 2016). - Istanbul, Turkey, 5-7 October 2016. URL: https://www.researchgate.net/publication/309291969_ Developing_a_Computer_Software_and_an_Android_Application For Bench Blasting (дата обращения: 12.02.2021).

20. $\bar{B}$ o-Hyun Kim, Mark K. Larson, Heather E. Lawson. Applying robust design to study the effects of stratigraphic characteristics on brittle failure and bump potential in a coal mine // International Journal of Mining Science and Technology. - 2018. - № 28. P. 137-144.

21. Уразаев Б., Исагулов С.Т. Роль буровзрывных работ при формировании качества руд и безопасности горных работ // Дальневосточная весна - 2020: материалы 18-й Международной научно-практической конференции по проблемам экологии и безопасности. - Комсомольск-на-Амуре: ФГБОУ ВО «КнАГУ», 2020. - С. 110-112.

Поступила: 17.03.2021 2.

\section{Информация об авторах}

Масаев Ю.А., кандидат технических наук, профессор кафедры строительства подземных сооружений и шахт Кузбасского государственного технического университета имени Т.Ф. Горбачева.

Масаев B.Ю., кандидат технических наук, доцент кафедры строительства подземных сооружений и шахт Кузбасского государственного технического университета имени Т.Ф. Горбачева.

Дрозденко Ю.В., кандидат технических наук, доцент, заведующий кафедрой строительства подземных сооружений и шахт Кузбасского государственного технического университета имени Т.Ф. Горбачева; доцент филиала Кузбасского государственного технического университета имени Т.Ф. Горбачева в г. Прокопьевске.

Аксенова O.Ю., кандидат технических наук, доцент, заведующая кафедрой начертательной геометрии и графики Кузбасского государственного технического университета имени Т.Ф. Горбачева. 


\title{
CONDITIONS OF STRESS WAVE FORMATION DURING BLASTING OF BOREHOLE CHARGES AND THEIR INFLUENCE ON ROCK FRACTURE QUALITY
}

\author{
Yuriy A. Masaev 1 , \\ myua.spssh@kuzstu.ru
}

\author{
Vladislav Yu. Masaev1, \\ masaevvu@kuzstu.ru
}

Yuriy V. Drozdenko1,2, duv.gmik@kuzstu.ru

\author{
Olesya Yu. Aksenova ${ }^{1}$ \\ aka.gp061@kuzstu.ru \\ 1 T.F. Gorbachev Kuzbass State Technical University, \\ 28, Vesennyaya street, Kemerovo, 650000, Russia. \\ 2 Branch of T.F. Gorbachev Kuzbass State Technical University in Prokopyevsk, \\ 19a, Nogradskaya street, Prokopyevsk, 650000, Russia.
}

The relevance. Explosive mining requires destroying rocks that lie at different depths in different mining and geological conditions and have different physical and technical properties. Explosion of explosive blast charges generates stress waves and pressure of explosion products, which have different effects on rocks of different hardness and to improve their action, explosive blast charges must be blasted with different deceleration.

The main aim of the research is to take into account the energy characteristics of explosives, the position of borehole charges relative to the natural fracturing of the rock to improve the quality of fracture of blasted rock.

Objects: underground construction facilities, coal and ore mines, mining enterprises for open-pit and underground mining.

Methods. To achieve this goal, we used field studies to establish the dependence of rock fracture quality on the operating time of bore charges, as well as analytical studies that allowed us to determine the values of the limiting radii of expansion of the charge cavity for some types of explosives.

Results. The results of the research are a more complete accounting of energy characteristics of explosives, the optimal volumetric position of borehole charges in relation to systems of natural fracturing of the environment, their correlation with the hardness of rocks, the cross section of mine workings and the depth of placing explosives charges, leading to creation of rational volumes of cuts, providing the highest performance blasting operations.

\section{Key words:}

Stress waves, explosive detonation, fracturing, fractured boreholes, short deceleration, borehole utilization factor, non-electric initiation system, primer-detonator.

\section{REFERENCES}

1. Masaev Yu.A., Masaev V.Yu. Study of the conditions for forming zones crack formation in the rock mass at construction of mine workings with the use of blasting. Industrial safety, 2020, no. 1, pp. 17-22. In Rus.

2. Pershin V.V., Masaev Yu.A., Milberger N.V. Investigation of the regularities of the formation of a pressure pulse in the charging cavity. Izvestiya vysshikh uchebnykh zavedenii. Gorny zhurnal, 2015, no. 1, pp. 93-102. In Rus.

3. Zhang Zong-Xian, Qiao Yang, Chi Li Yuan, Hou De-Feng. Experimental study of rock fragmentation under different stemming conditions in model blasting. International Journal of Rock Mechanics and Mining Sciences, 2021, no. 143. Available at: https://www.researchgate.net/publication/351266679_Experimenta 1_study_of_rock_fragmentation_under_different_stemming_condit ions_in_model_blasting (accessed: 12 February 2021).

4. Maslovsky A.N., Lukyanov A.N. Relevance of drilling and blasting operations with direct cuts when carrying out horizontal rock and exploration excavations with influence of geological and structural features of the rock massif. Bulletin of the Tomsk Polytechnic University, 2014, vol. 325, no. 1, pp. 166-172. In Rus.

5. Sarkar K., Singh T.N., Reddy D.V. Prediction of strength parameters by dynamic wave. Int J Earth Sci Eng., 2009, no. 2 (1), pp. 12-19.

6. Saikat Kuilia, Vedala Rama Sastry. A numerical modelling approach to assess the behaviour of underground cavern subjected to blast loads. International Journal of Mining Science and Technology, 2018, no. 28, pp. 975-983.

7. Grigorovskiy I. Using GGIS in the design of drilling and blasting operations in open pit mining. Zoloto $i$ tekhnologii, 2018, no. 2 (40), pp. 50-53. In Rus.

8. Masaev Yu.A., Karasev V.A., Sayapin V.V. Novye sredstva initsiirovaniya promyshlennykh zaryadov $\mathrm{VV}$ [New means of initiation of industrial explosive charges]. VIII Mezhdunarodnaya nauchno-prakticheskaya konferentsiya. Bezopasnost zhiznedeyatelnosti predpriyatiy $v$ promyshlenno razvitykh regionakh [VIII International scientific and practical conference. Safety of life of enterprises in industrially developed regions]. Kemerovo, KuzGTU Publ., 2009. pp. 173-175.

9. Masaev Yu.A., Domanov V.P. Electronic deceleration detonators. Industrial application experience. Bulletin of the Kuzbass State Technical University, 2010, no. 2, pp. 102-106. In Rus.

10. Isheyskiy V., Sanchidrián J.A. Prospects of applying MWD technology for quality management of drilling and blasting operations at mining enterprises. Minerals, 2020, no. 10. DOI: https://doi.org/10.3390/min10100925

11. Lukyanov V.G., Komashchenko V.N., Shmurygin V.A. Vzryvnye raboty [Imploding works]. Tomsk, TPU Publ., 2008. 404 p.

12. Kozlovskiy E.A., Sharov G.N., Kontorovich A.E., Gritsko G.I., Kuznetsov F.A., Kurlenya M.V., Kovalev V.A., Rostovtsev V.I., Belozerov I.M., Chernook V.A., Minin V.A., Vashlayeva N.Yu. Explosion hazard of gas in underground coal mining in Kuzbass. 
Fundamentalnye i prikladnye voprosy gornykh nauk, 2018, vol. 5, no. 1, pp. 76-82. In Rus.

13. Ganopolsky M.I., Baron V.L., Belin V.A., Pupkov V.V., Sivenkov V.I. Metody vedeniya vzryvnykh rabot. Spetsialnye vzryvnye raboty [Blasting techniques. Special blasting operations]. Moscow, MSMU Publ., 2007. 563 p.

14. Perepechaev I.F., Kravchenko A.N., Bashirov A.V. Improvement of drilling and blasting operations in the quarries of the association. Gorny zhurnal, 2014, no. 6, pp. 28-31. In Rus.

15. Lyashenko V.I., Khomenko O.E., Andreyev B.N., Golik V.I. Justification of drill and blast pattern designs for ore treatment before in-situ leaching. Mining informational and analytical bulletin (scientific and technical journal), 2021, no. 3, pp. 58-71. In Rus.

16. Ping Zhang, Ering Nordlund, Graham Swan, Changping Yi. Velocity amplification of seismic waves through parallel fractures near a free surface in fractured rock: a theoretical study. Rock Mechanics and Rock Engineering, 2019, no. 52, pp. 199-213.

17. Andreyev V.V., Timoshin I.V. Osobennosti vzryvaniya neelektricheskoy sistemoy SINV [Features of blasting by a nonelectric system of SINV]. Explosion technology, 2007, no. 97/54, pp. 131-134. Available at: https://sbornikvd.ru/vd_09754/index. html (accessed 12 February 2021).

18. Kotin S.V., Parkhomenko D.M., Pronin V.V., Bervin A.V. Implementation of the program for conducting experimental explosions in the branches of JSC MC Kuzbassrazrezugol for the period 2020-2021. Russian mining industry, 2020, no. 5, pp. 4446. In Rus.

19. Alaattin Sakcali, Huseyin Yavuz, Halim Cevizci. Developing a computer software and an android application for bench blasting. $6^{\text {th }}$ International Conference on Computer Applications in the Mineals Industries (CAMI 2016). Istanbul, Turkey, 5-7 October 2016. Available at: https://www.researchgate.net/publication/ 309291969_Developing_a_Computer_Software_and_an_Android _Application_For_Bench_Blasting (accessed: 12 February 2021).

20. Bo-Hyun Kim, Mark K. Larson, Heather E. Lawson. Applying robust design to study the effects of stratigraphic characteristics on brittle failure and bump potential in a coal mine. International Journal of Mining Science and Technology, 2018, no. 28, pp. 137144.

21. Urazaev B., Isagulov S.T. Rol burovzryvnykh rabot pri formirovanii kachestva rud i bezopasnosti gornykh rabot [The role of drilling and blasting operations in the formation of the quality of ores and the safety of mining operations]. Dalnevostochnaya vesna - 2020: materialy 18-y Mezhdunarodnoy nauchnoprakticheskoy konferentsii po problemam ekologii $i$ bezopasnosti [Far Eastern Spring - 2020: materials of the $18^{\text {th }}$ International Scientific and Practical Conference on Ecology and Safety]. Komsomolsk-on-Amur, KnASU Publ., 2020. pp. 110-112.

Received: 17 March 2021.

\section{Information about the authors}

Yuriy A. Masaev, Cand. Sc., associate professor, T.F. Gorbachev Kuzbass State Technical University.

Vladislav Yu. Masaev, Cand. Sc., associate professor, T.F. Gorbachev Kuzbass State Technical University.

Yuriy V. Drozdenko, Cand. Sc., head of Construction of Underground Structures and Mines Department, T.F. Gorbachev Kuzbass State Technical University; associate professor, Branch of T.F. Gorbachev Kuzbass State Technical University in Prokopyevsk.

Olesya Y. Aksenova, Cand. Sc., head of Descriptive Geometry and Graphics Department, T.F. Gorbachev Kuzbass State Technical University. 